\title{
The outbreak of Coronavirus Disease 2019: update on the clinical characteristics and treatment strategies.
}

\author{
kailiang $\mathrm{xu}^{1}$, huayong $\tan ^{1}$, yingfeng $\mathrm{fu}^{1}$, Qian wang ${ }^{1}$, shenbo han ${ }^{1}$, bo $\operatorname{tang}^{1}$, maojun $\mathrm{di}^{1}$, \\ and Qiang Tang ${ }^{2}$
}

${ }^{1}$ Affiliation not available

${ }^{2}$ Taihe Hospital

June 2, 2020

\begin{abstract}
In December 2019, with the outbreak of the unidentified pneumonia, a novel coronavirus was confirmed to be accountable for this disease and aroused great attention around the entire world. The World Health Organization (WHO) officially named the novel coronavirus as SARS-CoV-2 and the pneumonia of unknown origin as Corona Virus Disease (COVID-19). At present, COVID-19 has become an unprecedented global health crisis, with over 6 million confirmed cases and 350,000 deaths worldwide. The main manifestations are usually fever, cough, fatigues, breathlessness, and among others. To date, specific antiviral agents against the SARS-CoV-2 are not available. Extensive measures to reduce person-to-person transmission of COVID-19 have been implemented to control the current outbreak. In this review, we detail current evidence and understanding of the etiology, epidemiology, clinical characteristics, treatment of COVID-19, which may provide a reference for future studies and help in the prevention and control of the COVID-19 epidemic.
\end{abstract}

\section{Introduction}

In December 2019, an outbreak of unidentified pneumonia characterized by fever, dry cough, and fatigue happened in Wuhan, Hubei, China (Zhu et al., 2020). With the spread of the disease, the number of infected patients increased substantially which has become the most challenging health emergency all over the world. Sequence analysis of the coronavirus has shown a structure typical to that of other coronaviruses such as SARS coronavirus and MERS coronavirus (N. Chen et al., 2020). It also revealed that the new coronavirus has the smallest genetic distance from bat coronavirus, and about $80 \%$ similarity with SARS-CoV, and $50 \%$ similarity with MERS-CoV (P. Sun, Lu, Xu, Sun, \& Pan, 2020). Thereafter, the coronavirus was designated as SARS-CoV-2 and the infectious disease was named Coronavirus Disease 2019 (COVID-19) by the World Health Organization (WHO) (Li-Li et al., 2020; Sohrabi et al., 2020; Zhu et al., 2020). Besides, the nosocomial transmission was detected on January 20, 2020, which suggested that COVID-19 can be transmitted from human to human.

Due to the effective prevention and control measures, the epidemic has been brought under control in China, South Korea, Japan, and more than $90 \%$ of patients have recovered and discharged in these countries. However, the confirmed cases are still growing rapidly in American, European and African countries including the United States, Brazil, Russia, the UK and South Africa (WHO, 2020a). As experience in recognition, diagnosis, and treatment of COVID-19 builds, WHO has launched global megatrial of the four most promising coronavirus treatments (WHO, 2020b). The present article is to provide a review of the characteristics of COVID19, including the epidemiology, etiology, clinical features, pathological changes, and treatment, and the experience of prevention measures for this disease.

\section{Epidemiology ofCOVID-19}


Since December 2019, the first 27 cases of unidentified pneumonia were reported by the Wuhan Municipal Health Commission (Ashour, Elkhatib, Rahman, \& Elshabrawy, 2020). On January 11, 2020, the pathogen of the pneumonia was initially confirmed as a novel coronavirus. On January 20, 4 confirmed cases of COVID19 were reported from three countries outside of China including Thailand (2 cases), Japan (1 case), and the Republic of Korea (1 case). As the epidemic expanded, daily confirmed cases increased to 14,109, before falling to 1748 on February 18. And since then, the number of daily emerging cases gradually falls below 1000 for the first time in China. However, the number of confirmed cases reported daily increased rapidly abroad. Data released on February 25, 2020 showed foreign countries had overtaken China in confirmed cases per day for the first time. And then the disease spread rapidly in Europe and in the United States (Figure 1a). Up to this point, there have been 84,543 confirmed cases in China and 6,218,350 cases were confirmed in 212 countries outside of China with 370,100 fatalities(Figure 1b) . Recently, the five countries with the greatest number of patients analyzed were the United States $(1,839,679$ cases, 106,261 deaths, casefatality rate: 5.8\%), Brazil (514,992 cases, 29,341 deaths, case-fatality rate: 5.7\%), Russia (414,878 cases, 4855 deaths, case-fatality rate: $1.03 \%$ ), Spain (286,509 cases, 27,127 deaths, case-fatality rate: 9.5\%), and the United Kingdom (274,762 cases, 38,489 deaths, case-fatality rate: 14.0\%) (WHO, 2020a) (Figure 1c) . Since the first case of COVID-19 was reported in the U.S., the numbers of confirmed cases and deaths increase exponentially with steady growth at 20,000-30,000 per day. Though the outbreak of COVID-19 appears at later stage in Russia and Brazil, the number of confirmed cases has exceeded most European and American countries. From November 2019 to May 25, 2020, the number of cumulative deaths caused by the COVID-19 was 350,057, and the overall case-fatality rate (CFR) was $6.3 \%$ which was lower than that of the SARS (9.60\%) and MERS (34.4\%) (WHO, 2020a)(Figure 1d, Table 1) .

The transmission of infectious diseases must rely on three requirement conditions: sources of infection, routes of transmission, and susceptible hosts. A growing body of scientific evidence suggests that COVID-19 is a zoonotic disease as with SARS and MERS, and originated from the wild bat (Evans, 2013; Keeling \& Rohani, 2011). Though the strands of evidence suggest the possibility of an initial zoonotic emergence, the role of intermediate hosts such as pangolins, snakes, turtles, hamster, ferret, and other wild animals in the origin of SARS-CoV-2 still remains controversy (Tiwari et al., 2020). Close contact with symptomatic cases and asymptomatic cases with silent infection are the main transmission routes of 2019-nCoV infection. It suggested that SARS-CoV-2 can be transmitted through respiratory aspirates, droplets, contacts, and digestive tract transmission that remained to be confirmed (Peng et al., 2020). Vertical transmission was sporadically reported in some media but not yet proved(H. Chen et al., 2020). Reports showed that the basic reproductive values (R0) of COVID-19 were calculated between 1.5 and 3.5 (Eisenberg, 2020; Natsuko Imai, 2020). Therefore, SARS-CoV-2 appears to be more infectious than SARS-CoV or MERS-CoV based on R0 values at the early stage of this outbreak (Ying, A, Annelies, \& Joacim, 2020) (Table 1. ). Similar to SARS and MERS, nosocomial transmission was a severe problem. COVID-19 has posed a difficult challenge to healthcare facilities from both the impact of healthcare-associated transmission and the resource burden of controlling and preventing further spread. It has been reported that a total of 3,019 health workers were infected, accounting for $4.17 \%$ of total cases (Epidemiology Working Group for Ncip Epidemic Response \& Prevention, 2020). In terms of susceptible populations, people are generally susceptible to COVID-19 regardless of age or gender. And the elderly and those with underlying chronic diseases (hypertension, diabetes, COPD) are more likely to become severe cases. A retrospective cohort study by Chao et al. also demonstrated that patients with hypertension and diabetes accounted for 20-30\% of total infected patients and had a higher risk of developing into acute respiratory distress syndrome (ARDS) or multiple organ failure, and have a higher case fatality rate than those without (Chaomin, 2020). The ACE2 receptor, an important member of the renin-angiotensin system (RAS), is highly expressed in the cardiovascular/cerebrovascular and lung tissue in the hypertension patients (Kristensen et al., 2015). Current reported data revealed that $86.6 \%$ of confirmed patients were aged $30-79$ years and $1-2 \%$ of the patients were children and newborns (1.5 months to 17 years). The clinical course of pregnant women with COVID-19 is similar to patients of the same age (Schmid \& Fontijn, 2020).

\section{The etiology of COVID-19}


Coronavirus is comprised of single-stranded positive RNA virus that belongs to an order Nidovirales , family Coronaviridae, and subfamily Orthocoronavirinae (Jie, Fang, \& Zheng-Li, 2019). Coronavirus can be divided into four genera: $\alpha-, \beta-, \gamma-, \delta$-coronavirus according to the characteristics of serotype and genome $(\mathrm{P}, \mathrm{Xin}, \mathrm{P}, \& \mathrm{Y}, 2019)$. Genome sequences analysis showed that the novel coronavirus is a new type of coronavirus (SARS-CoV-2) and belongs to the $\beta-\mathrm{CoV}$ genera (Li-Li et al., 2020; Ren et al., 2020). Genetics researchers and scientists worldwide found that the genomes of SARS-CoV-2 have naturally evolved, thereby rejecting a hypothesis of a possibility of recent recombination event as the cause of this outbreak (X. Xu et al., 2020). Since its genome is similar to bat coronavirus, scientists conjecture SARS-CoV-2 is most likely to be originating from the bat (Cui, Li, \& Shi; Rothan \& Byrareddy, 2020). Virion spike (S) protein is the key components which determines the host tropism of the virus. S protein is composed of two subunits: S1 and S2, which are responsible for the receptor binding and fusion of virus with cell membrane respectively (Shajahan, Supekar, Gleinich, \& Azadi, 2020). Current studies have revealed that SARS-CoV-2 shared the same receptors with SARS-CoV and MERS-CoV for invading the host cells, and the angiotensin-converting enzyme 2 (ACE2) receptor has been identified as the main cell entry receptor of ARS-CoV-2 (Kuba et al., 2020). And structural and biophysical analysis revealed that the affinity of SARS-CoV-2 for ACE2 is 10 to 20 times higher than that of the SARS-CoV (Xie \& Chen, 2020). A vitro study confirmed that SARSCoV-2 can survive in human respiratory epithelial cells for 96 hours in vitro (Yu, 2020). SARS-CoV-2 also shared the same physical and chemical characteristics with SARS-CoV and MERS-CoV. Coronaviruses are hypersensitive to ultraviolet rays and heat. And it can be killed easily by exposed to 56 for $30 \mathrm{mins}, 75 \%$ ethanol, chlorine disinfectant, peracetic acid, and chloroform (Deng \& Peng, 2020).

\section{Clinical characteristics ofCOVID-19}

\subsection{Clinical manifestations}

Based on the current epidemiological investigation, the incubation period of the COVID-19 is about 1-14 days (Linton et al., 2020). It has been reported that the incubation period for severe cases may be shorter than that of the mild cases and it tended to be shorter among patients older than 60 than those aged less than 60 years old. The most common symptoms at onset of COVID-19 illness according to WHO report that was done on more than 70,000 cases in China are as following: fever (88\%), cough (68\%), and fatigue (38\%), dyspnea (21.9\%) (Chappell, 2020; Pranab, Nazia, Anup, Bhabatosh, \& Raman, 2020). Non-respiratory symptoms like nausea, vomiting, and diarrhea were uncommon presentations (Luigi Angelo, Giovanni, \& Giacomo De, 2020). Infected children might appear asymptomatic or present with fever, dry cough, and fatigue, and few presented with gastrointestinal symptoms including abdominal discomfort, nausea, vomiting, abdominal pain, and diarrhea (K. Shen et al., 2020). According to the report on "Diamond Princess", 189 $(17.9 \%)$ patients were asymptomatic carriers (Lee, 2020), which indicated that there exist a large number of asymptomatic individuals remain undiscovered in the community. It is crucial to identify and isolate asymptomatic carriers and mild patients to contain the outbreaks in later stages.

According to the severity of symptoms, COVID-19 can be divided into four classes: mild, moderate, severe, and critical types (Zu et al., 2020). Mild patients presented low-grade fever, mild fatigue or dry cough only. Moderate patients are always characterized by fever, respiratory symptoms, and radiographic abnormality. The severe patients should meet one of these criteria: A. dyspnea B. $\mathrm{PaO} 2 / \mathrm{FiO} 2<300 \mathrm{mmHg}$. C. Oxygen saturation $<93 \%$ in ambient air (Z. Sun, Thilakavathy, Kumar, He, \& Liu, 2020). And about one-third of the severe patients may develop into critically ill with acute respiratory distress syndrome, septic shock, and coagulation dysfunction within a week (Gorbalenya, 2020). According to the recent survey conducted by the China CDC, $80.9 \%$ of confirmed patients were mild pneumonia cases, $13.8 \%$ were severe cases, and $4.7 \%$ were the critical cases (Surveillances, 2020).

\subsection{Laboratory examination}

In the early stage of the COVID-19, the white blood cell count is normal or decreased, with decreased lymphocyte count. The lymphocyte subtypes were further analyzed and found that the count of CD3+, $\mathrm{CD} 4+$ and CD8 $+\mathrm{T}$ cell in the severe patients was significantly lower than that of the non-severe group 
(Xiaobo et al., 2020). Some patients may present elevated levels of liver enzymes (TBIL DBIL ALB ALT AST), lactate dehydrogenase, C-reactive protein (CRP), and coagulation disorders (D-dimer, fibrinogen) (D. Wang et al., 2020). The level of cardiac troponin I, N-terminal pro-brain natriuretic peptide and creatinine may be increased in severe and critically ill patients, which indicated that COVID-19 could induce myocardium, kidneys, and liver injury directly or indirectly (Jin et al., 2020). Critical patients often have a higher level of inflammatory factors such as IL2, IL6, IL7, IL10, and TNF- $\alpha$ (Chaolin et al., 2020). Chen et al. reported that elevated levels of C-reactive protein, D-dimer, and procalcitonin were associated with severe patients compared to non-severe patients (C. Huang et al., 2020). Zhao et al. found that a higher level of D-dimer, high-sensitivity cardiac troponin I, serum ferritin, lactate dehydrogenase, and IL-6 were the independent risk factors of death (Chaomin, 2020).

Nucleic acid testing is the standard method for the diagnosis of COVID-19 infections (Udugama et al., 2020). Based upon the evidence from clinical research, the SARS-CoV-2 nucleic acids can be detected in nasopharyngeal swabs, sputum, lower respiratory tract secretions using reverse transcription-polymerase chain reaction (RT-PCR) method (Rothan \& Byrareddy, 2020). RT-PCR has been widely deployed in diagnostic virology and has yielded few false-positive outcomes. However, some research suggested that this method usually showed false-negative results due to poor RNA stability, the quality of the kit and specimen (Kimberly E. Hanson, 2020; Udugama et al., 2020). Both IgM and IgG titers were markedly increased in nearly all patients, which was normally considered as a transition from earlier to later period of infection (Zhang et al., 2020). Therefore, the combination of RT-qPCR and IgM/IgG antibody assay may be useful for diagnosing COVID-19 and screening of SARS-CoV-2 carriers, symptomatic or asymptomatic (Imai et al.; Z. Li et al., 2020).

\subsection{Radiographic imaging}

Radiological examinations are of great importance in the early detection and management of COVID-19. According to current experience, lung imaging manifests can be found earlier than clinical symptoms, so imaging examination is vital in preclinical screening (Sohrabi et al., 2020). Therefore, the suspected cases should undertake chest examination as soon as possible.

In the early stage, multiple small patchy shadows and interstitial changes were detected in the extrapulmonary zone. And then, it developed into multiple ground-glass infiltrations (Dawei Wang et al., 2020). In severe and critically cases, lung lesions usually involved most commonly $4-5$ lobes in the bilateral lower and upper lobes. The first report of COVID-19 patients described that bilateral lung involvement was detected in $80 \%$ of patients, and consolidative pattern changes were always observed in most patients in an intensive care unit (ICU), but ground-glass pattern always showed in patients not in the ICU (Bernheim et al., 2020). Shi et al. analyzed the CT changes and found that most patients even the asymptomatic patients showed dynamic changes from focal unilateral to diffuse bilateral ground-glass opacities and then progressed to consolidations within 1-3 weeks (X. W. Xu et al., 2020). In general, combining assessment of imaging features with clinical and laboratory findings can facilitate the diagnosis of COVID-19 pneumonia and evaluate the severity of the disease.

\section{Thepathological changes in COVID-19 patients}

Pathological examination of patients with COVID-19 would confirm laboratory and radiological findings and contribute to further study and a better understanding of mechanisms of the disease (Z. Xu et al., 2020). A recent study reported the biopsy results from two patients who underwent surgery for malignancy and then were found to have been infected with SARS-CoV-2, which provided first opportunities to study the pathology of COVID-19. It revealed that the lungs of patients exhibited edema, proteinaceous exudate, focal reactive hyperplasia of pneumocytes with patchy inflammatory cellular infiltration, and multinucleated giant cells, but hyaline membranes were not prominent (Tian et al., 2020). This study may describe early phase changes of the lung pathology of COVID-19 pneumonia. After that pathologist from Huazhong University of Science and Technology, Wuhan, China performed autopsy from 12 dead patients and the results were released by the national health commission, China (Wang, Du, Yue, \& Chen, 2020). The histopathological 
changes for different organs are summarized below:

Lung The lungs showed evident multi-pulmonary consolidation, acute interstitial inflammatory infiltrates and congestion in the alveolar septae. The lumina of alveoli and bronchioles were variably filled with protein-rich edema fluid, erythrocytes, cellular debris, and lymphocytes. Type II alveolar epithelial cells proliferated obviously with inclusion bodies inside. The blood vessel of the alveolar septum had congestion and edema, in which the infiltration of lymphocytes and monocytes, and intravascular hyaline thrombosis can be seen. Focal hemorrhage and necrosis of the lung tissue caused hemorrhagic infarction. Diffuse interstitial pulmonary fibrosis would be presented with the disease progress. Bronchial epithelial cells were degenerated, necrosis and fell off. Mucus plugs were visible in the bronchial lumen. Due to the over-inflation of the alveoli, a small number of the alveolar septum was broken, or the cysts were formed. SARS-CoV-2 particles could be observed in the cytoplasm of bronchial mucosal epitheliums and type II alveolar epithelial cells under an electron microscope. Immunohistochemical staining showed that some alveolar epitheliums and macrophages were positive for SARS-CoV-2 antigens. These tissue types also tested positive for nucleic acids on RT-PCR.

Immune system The volume of the spleen decreased significantly and the number of lymphocytes was significantly reduced. There were focal patchy hemorrhages, necrosis and proliferation, and phagocytosis of macrophages in the splenic tissue, with atrophy of white pulp lymphoid aggregates. The number of lymphocytes decreased obviously, and necrosis was visible in lymph nodes. Immunohistochemical staining showed that CD4 $+\mathrm{T}$ and CD8 $+\mathrm{T}$ cells were reduced in the spleen and lymph nodes. The number of three cell lines in the bone marrow was reduced.

Cardiovascular system There was notable degeneration and necrosis in the myocardial cells, and a few monocytes, lymphocytes and neutrophils infiltrated in the interstitium. Endothelial shedding, endovascular inflammation and thrombosis were visible in the blood vessel.

Liver and gallbladder The volume of the liver increased and its color was dark red. There were degeneration of hepatocytes, congestion of hepatic sinus, focal necrosis with neutrophil infiltration and microthrombosis, which feature the repeated interchange of these kinds of pathological course. The gallbladder was filled with bile.

Kidney There was proteinaceous exudate in the glomerular cavity and degeneration and necrosis in renal tubular epitheliums. Hyperemia, microthrombus and focal fibrosis can be observed in the renal interstitium.

Further, a cohort study conducted by Dominic Wichmann from Intensive Care Medicine University Medical Center Hamburg, demonstrated that $4(4 / 12,33.3 \%)$ patients died of pulmonary embolism, and $8(8 / 12$, $66.7 \%$ ) exhibited substantial histomorphological diffuse alveolar damage. This study revealed that SARSCoV-2 RNA could be detected at high concentrations in the lung of all the patients. And more than half of the patients demonstrated high viral RNA titers SARS-CoV-2 RNA was detected in the liver, kidney, or heart (Wichmann et al., 2020).

\section{Treatment of COVID-19}

Patients should be allocated to designated treatment areas according to the disease severity. Confirmed and suspected patients should be isolated in hospitals with applicable protective equipment and special conditions (e.g. negative pressure ward.). The confirmed cases can be admitted in the same ward, but the suspected cases would be better isolated in a single room to avoid transmission. Besides, critical cases should be treated in ICU as soon as possible (N. H. C. S. A. o. T. C. Medicine, 2020).

Until now, no medicine or anti-virus vaccine has yet been officially recommended for COVID-19 infection (J. Sun et al., 2020). In fact, many countries are focusing on investigating specific medicine to control the infections of SARS-CoV-2 such as vaccines, antibodies, and interferon, which may require a long time of clinical trial (Amanat \& Krammer, 2020). Currently available treatment approaches include symptomatic and supportive therapies including supplementary oxygen, mechanical ventilation, glucocorticoids and infection prevention and control (Jin et al., 2020). Antibiotics have no role in treating COVID-19 patients, but they can be used in the case of a secondary bacterial infection. As for antiviral therapy, several agents previously 
used to treat SARS and Middle East Respiratory Syndrome (MERS) and HIV have been considered as the most potential candidates for COVID-19 patients in China firstly (Kang et al., 2020). With the spread of COVID-19, a large number of pre-clinical and clinical studies all over the world have been underway to investigate the potential agents for COVID-19.

Lopinavir/Ritonavir were the first two antivirals extensively applied for treatment of the COVID-19 in china. Though lopinavir/ritonavir have been used for the treatment of human immunodeficiency virus (HIV) infection with a generally good safety profile, clinical feasibility and safety require further investigation (Kaplan \& Hicks, 2005). As has been pointed out, lopinavir/ritonavir usually has compromised interactions with many drugs commonly used in severe patients, which made it less promising for the treatment of COVID-19 patients. Nevertheless, this antivirus has been recommended by the available guidelines in many other countries including the US, Japan (Cao et al., 2020; Stower, 2020). Favilavir was another anti-virus drug that has been effective in relieving the symptoms COVID-19 patients in Japan and China (Abd El-Aziz \& Stockand, 2020; Elfiky, 2020). However, this antivirus is not currently approved by the Food and Drug Administration (FDA) of the US (Guan et al., 2020; Li G Fau - De Clercq \& De Clercq, 2020).

Remdesivir, a monophosphoramidate prodrug of an adenosine analogue, could inhibit viral replication through incorporating into nascent viral RNA chains resulting in pre-mature termination (M. Wang et al., 2020). A variety of studies have confirmed that remdesivir showed a significant antiviral activity against a broad variety of RNA viruses including SARS-CoV, MERS-CoV and Ebola virus in cultivated cells, mice and non-human primates (NHP) models (de Wit et al., 2020; Warren et al., 2020; Xie \& Chen, 2020). Therefore, remdesivir has emerged as the most promising candidate based on the broad antiviral spectrum for the treatment of SARS-CoV-2 infection (Marto \& Monteiro, 2020). Meanwhile, a large number of randomized clinical trials have been strictly conducted to investigated its antiviral and safety profile in patients with COVID-19 such as: NCT04252664 and NCT04257656 in many countries (Esposito, Noviello, \& Pagliano, 2020; Y. Wang et al., 2020). Recently, a randomized, double-blind clinical trial conducted in China revealed that remdesivir could not improve the time to clinical cure and elimination of viruses, as well as the mortality in patients with COVID-19 (Y. Wang et al., 2020). However, the preliminary clinical study of compassionate use of remdesivir released in England Journal of Medicine came to diametrically opposed conclusions, which demonstrated that $68 \%(36 / 53)$ of patients showed significant clinical improvement with a mortality rate of 18\% (Grein et al., 2020). Similarly, the National Institutes of Health of the US reported the findings from a randomized, placebo-controlled trial of remdesivir among 1,063 patients, which suggested that patients who received remdesivir tend to improve faster than patients without, and remdesivir could significantly reduce the mortality rates. Therefore, additional studies with the multicenter and large samples are required to evaluate the safety and efficiency of this antiviral agent.

Chloroquine (CQ) and hydroxychloroquine (HCQ), belonging to the class of aminoquinolines, have been approved for the treatment of malaria, chemoprophylaxis, and autoimmune diseases including lupus and rheumatoid arthritis (Jakhar \& Kaur, 2020; Rempenault et al., 2020). In addition, these small-molecule agents have been identified as a promising broad-spectrum antiviral. Previous studies have shown that CQ and HCQ could inhibit the activity of RNA virus such as HIV, HCV, SARS, MERS, Ebola, Hendra viruses in vitro via targeting endosomal acidification as the major determinant of antiviral activity and affecting glycosylation of ACE2 receptor that are required for viral entry (Costanzo, De Giglio, \& Roviello, 2020; Lentini, Cavalluzzi, \& Habtemariam, 2020; Pereira, 2020; Savarino A Fau - Di Trani, Di Trani L Fau Donatelli, Donatelli I Fau - Cauda, Cauda R Fau - Cassone, \& Cassone, 2020). A recent study also supported the potent activity of CQ and HCQ against SARS-CoV-2, which suggesting the inhibiting effect of viral replication during the initial phases of viral infection. Therefore, they have been approved by the FDA and currently recommended for the treatment of COVID-19 patients. The China National Health Commission reported that CQ may be one of the three drugs with a promising profile for treatment of SARS-CoV-2, but not for infection prevention due to the frequent side effects including severe nausea, polymorphic ventricular tachycardia, digestive disorders, long QT syndrome, and increased risk for sudden death (Juurlink, 2020). A France study among 36 patients reported that single-agent HCQ or combination with azithromycin therapy holds the promise of shortening the clearance of SARS-CoV-2 RNA in the upper respiratory tract (CHEN 
Jun, 2020). Another report with a larger trial of 84 patients treated with hydroxychloroquine and 97 cases for control suggested that there was no evidence of improvement in patient outcomes between the two groups (Marto \& Monteiro, 2020). According to the latest report released by European Medicines Agency (EMA) in April, CQ and HCQ should be only used in clinical trials or emergency use programs (Ferner \& Aronson, 2020). Therefore, they are currently presented as an option for the treatment of hospitalized COVID-19 patients in several countries, including the United States, Portugal, Brazil, and France (Health., 2020; P. S. o. c. medicine, 2020; Wilson KC, 2020).

According to the previous reports, SARS-CoV-2 induced aberrant immune responses and excessive infection cytokine storm with increased plasma concentrations of interleukins IL-6, IL-2, IL-7, and IL-10 and tumor necrosis factor are believed to play major roles in disease severity (Yang et al., 2020). Zhou et al confirmed that the value of IL-6 was significantly elevated in patients with ARDS who died compared with patients who survived(Wu et al., 2020). Tocilizumab, a humanized IL-6-receptor (IL-6R) monoclonal antibody, has been experimentally administered in the treatment of COV-ID-19 patients with the severe form of the disease and elevated IL-6 levels in China and Italy (Commission., accessed 2020 Apr 12). In March, The Italian Medicines Agency (AIFA) announced the launch of the clinical phase 2 study named to evaluate the efficacy and safety of TCZ in the treatment of COVID-19 (Esposito et al., 2020). Up to now, many multicenter clinical trials have been registered and spanning the United States, Canada, China, and Europe. Therefore, the WHO has not given a positive response for the use of tocilizumab in COVID-19, due to insufficient evidence.

Convalescent plasma has been applied to treatment of infectious diseases for more than one century. Based on the initial successful experience in the treatment of SARS and MERS, convalescent plasma therapy may be another potentially promising option for COVID-19 patients by providing passive immunity against infectious agents (Cheng et al., 2020; van Griensven et al., 2020). Researches from China have demonstrated that convalescent plasma could significantly improve the symptoms and reduce the mortality rate (Duan et al., 2020; C. Shen et al., 2020). Recently, the FDA has approved convalescent plasma therapy for emergency use to treat COVID-19 patients especially patients with severe condition. Inevitably, convalescent plasma may increase the risks of blood-borne pathogen transmission such as HIV, HBV, HCV and so on (Bhimraj et al., 2020).

Corticosteroids have been previously used in SARS, MERS, H1N1 viral pneumonia with possible immunomodulatory properties (Arabi et al., 2020; Booth et al., 2020). However, reports have confirmed that instead of improving of the mortality among patients with SARS and MERS, corticosteroids increased risk of extended hospital lengths of stay, delayed viral clearance and secondary infections (Coondoo, Phiske, Verma, \& Lahiri, 2014). One study among 31 patients from China showed that corticosteroid use did not influence the outcome of patients with mild COVID-19 (Zha et al., 2020). And systematic use of corticosteroids is even higher in critically ill patients with ARDS. However, a multicenter study of 213 patients from the University of Maryland Medical Center demonstrated that low-dose glucocorticoid treatment during the early stages of COVID-19 could significantly reduce the likelihood of being admitted to ICU, the requirement for mechanical ventilation and mortality. Therefore, the treatment of COVID-19 with glucocorticoids is rather controversial, and the WHO has recommended against routinely administering systemic corticosteroids to patients with COVID- 19 .

\section{The physical and mental health of medical staff}

During the spread of the epidemic, COVID-19 prevention and control attracted considerable attention. The highly pathogenic and infectious COVID-19 undoubtedly poses a great challenge to the health of front-line medical staff who were testing for and treating patients with COVID-19 are at a higher risk of contracting it than the general publication (Magellan Health Insights, 2020). According to the existing study results, medical staffs were at high risk of infection during the MERS and SARS outbreaks, with $18.6 \%$ of MERS cases occurring in medical staff and $21 \%$ of SARS cases occurring in medical staff (Chan-Yeung, 2004; Kim et al., 2020). Due to insufficient understanding of the characteristics of pathogenic and insufficient and unequally-distributed medical resources at the beginning of the epidemic outbreak, a large number of 
medical staff were infected during the battle against COVID-19. According to the International Council of Nurses (ICN), there have been more than 90,000 health workers were infected, and 260 nurses died from this contagious disease (Nurses, 2020). Health care professionals, particularly those working in emergency units and resuscitation departments, were most often infected during the operation with high level of exposure levels such as endotracheal suction and intubation, nasogastric feed, cardiopulmonary resuscitation and high flow-rates of oxygen and so on (Gamage et al., 2005). In addition, many professionals may consistently experience a high intensity of work with the need of wearing Personal Protective Equipment (PPE), which usually cause physical discomfort and difficulty breathing (Huang, Han, Luo, Ren, \& Zhou, 2020; Shigemura, Ursano, Morganstein, Kurosawa, \& Benedek, 2020).

Apart from physical suffering, health professionals were suffered from great psychological pressure and other psychological-related problems. Frontline medical staff, especially those in the most severely affected city: New York, Madrid, London, are highly vulnerable to experiencing physical exhaustion, fear, emotion disturbance, sleep problem and concern for themselves, their families and colleagues (Kisely et al., 2020; Malta, Rimoin, \& Strathdee, 2020). In one study of 1563 health medical staffs, 50.7\% of the participants experienced serious depression symptoms, $44.7 \%$ anxiety, and $36.1 \%$ sleep disorders (Chersich et al., 2020). Another multicenter study conducted in Singapore and India demonstrated that $19.8 \%$ of healthcare workers exhibited varying degrees of psychological discomfort: moderate to very-severe depression, anxiety, psychological distress, and 32.3\% always experienced with headache, panic (Cullen, Gulati, \& Kelly, 2020). Early evidence indicates that medical staff especially the ones who participated in the treatment of SARS patients was more susceptible to psychological disorders. Therefore, healthcare organizations should adopt following key strategies urgently to protect the mental health of employees with their duty: enhanced decision making support to provide sufficient medical resources; the provision of time and space for clinicians to decompress; and staff working consistently in the same team (Kisely et al., 2020). At the same time, the National Health Commission of China (NHC) has integrated psychological crisis intervention into the general deployment of disease prevention, and released the clinical practice guideline of emergency psychological crisis interventions, psychological counseling for health staff. Recently, the platform for remote health counseling services has been established with psychological professionals in Wuhan, Macao and Rome, which made it easier and safer to provide mental health education for both patients and front-line medical staff (W. Li et al., 2020; Macau., 2020).

Therefore, we cannot ignore providing psychological counseling and crisis intervention training for medical staff. In addition, reasonable schedules, adequate sleep, and appropriate diet and exercise are also the keys to improving the body's immunity and reducing the risks of suffering.

\section{Conclusion}

The outbreak of COVID-19 before the Spring Festival of China, and the massive population flow has challenged the economic, medical and public health infrastructure of China and even all over the world. SARSCoV-2 can be transmitted from person to person and be more infectious than SARS-CoV or MERS-CoV. To date, there are no specific antiviral agents against the virus, and the most effective to prevention and control strategies are early identification, early isolation, early diagnosis, and early treatment. In addition, special attention and efforts to protect or reduce transmission should be applied in susceptible populations including children, elderly people, and health care providers. And appropriate psychological interventions and health education on knowledge for disease prevention and control may be necessary to reduce the risks of infection and heighten the work efficiency greatly. Finally, with the efforts of China and other countries, we will learn more about the epidemiology of the virus and develop specific vaccines and therapeutic drugs to eliminate the threat of COVID-19.

Acknowledgements: We thank Medjaden Bioscience Limited (HongKong, China) for proofreading this manuscript.

Conflict of interest: None declared.

Ethics Statement: The authors confirm that the ethical policies of the journal, as noted on the journal's 
author guidelines page, have been adhered to. No ethical approval was required as this is a review article with no original research data.

Funding statement: None.

Data availability statement: The data that support the findings of this study are available on request from the corresponding author. The data are not publicly available due to privacy or ethical restrictions.

\section{Reference:}

Abd El-Aziz, T. M., \& Stockand, J. D. (2020). Recent progress and challenges in drug development against COVID-19 coronavirus (SARS-CoV-2) - an update on the status.Infect Genet Evol, 83 (1567-7257 (Electronic)), 104327. doi: 10.1016/j.meegid.2020.104327

Amanat, F., \& Krammer, F. (2020). SARS-CoV-2 Vaccines: Status Report. Immunity, 52 (4), 583-589. doi: $10.1016 /$ j.immuni.2020.03.007

Arabi, Y. A.-O., Mandourah, Y., Al-Hameed, F., Sindi, A. A., Almekhlafi, G. A., Hussein, M. A., . . - Fowler, R. A. (2020). Corticosteroid Therapy for Critically Ill Patients with Middle East Respiratory Syndrome. (1535-4970 (Electronic)).

Ashour, H. M., Elkhatib, W. F., Rahman, M., \& Elshabrawy, H. A. (2020). Insights into the Recent 2019 Novel Coronavirus (SARS-CoV-2) in Light of Past Human Coronavirus Outbreaks. Pathogens, 9 (3), 186.

Bernheim, A., Mei, X., Huang, M., Yang, Y., Fayad, Z. A., Zhang, N., . . . Chung, M. (2020). Chest CT Findings in Coronavirus Disease-19 (COVID-19): Relationship to Duration of Infection. Radiology, 295 (3), 200463. doi: 10.1148/radiol.2020200463

Bhimraj, A., Morgan, R. L., Shumaker, A. H., Lavergne, V., Baden, L., Cheng, V. C., . . . Falck-Ytter, Y. (2020). Infectious Diseases Society of America Guidelines on the Treatment and Management of Patients with COVID-19. LID - 10.1093/cid/ciaa478 [doi] LID - ciaa478. (1537-6591 (Electronic)).

Booth, C. M., Matukas Lm Fau - Tomlinson, G. A., Tomlinson Ga Fau - Rachlis, A. R., Rachlis Ar Fau Rose, D. B., Rose Db Fau - Dwosh, H. A., Dwosh Ha Fau - Walmsley, S. L., . . . Detsky, A. S. (2020). Clinical features and short-term outcomes of 144 patients with SARS in the greater Toronto area. (0098-7484 (Print)).

Cao, B., Wang, Y., Wen, D., Liu, W., Wang, J., Fan, G., . . . Wang, C. (2020). A Trial of Lopinavir-Ritonavir in Adults Hospitalized with Severe Covid-19. N Engl J Med, 382 (19), 1787-1799. doi: 10.1056/NEJMoa2001282

Chan-Yeung, M. (2004). Severe acute respiratory syndrome (SARS) and healthcare workers. Int J Occup Environ Health, 10 (4), 421-427. doi: 10.1179/oeh.2004.10.4.421

Chaolin, H., Yeming, W., Xingwang, L., Lili, R., Jianping, Z., Yi, H., . . . Bin, C. (2020). Clinical features of patients infected with 2019 novel coronavirus in Wuhan, China. The Lancet (Pre-publis).

Chaomin, W. (2020). Risk Factors Associated With Acute Respiratory Distress Syndrome and Death in Patients With Coronavirus Disease 2019 Pneumonia in Wuhan, China.JAMA Internal Medicine.

Chappell, B. (2020). CORONAVIRUS: COVID-19 IS NOW OFFICIALLY A PANDEMIC.

Chen, H., Guo, J., Wang, C., Luo, F., Yu, X., Zhang, W., . . . Zhang, Y. (2020). Clinical characteristics and intrauterine vertical transmission potential of COVID-19 infection in nine pregnant women: a retrospective review of medical records.Lancet, 395 (10226), 809-815. doi: 10.1016/S0140-6736(20)30360-3

CHEN Jun, L. D., LIU Li, LIU Ping, XU Qingnian, XIA Lu, LING Yun, HUANG Dan, SONG Shuli, ZHANG Dandan, QIAN Zhiping, LI Tao, SHEN Yinzhong, LU Hongzhou. (2020). A pilot study of hydroxychloroquine in treatment of patients with common coronavirus disease-19 (COVID-19). 
Chen, N., Zhou, M., Dong, X., Qu, J., Gong, F., Han, Y., . . . Zhang, L. (2020). Epidemiological and clinical characteristics of 99 cases of 2019 novel coronavirus pneumonia in Wuhan, China: a descriptive study. Lancet, 395 (10223), 507-513. doi: 10.1016/S0140-6736(20)30211-7

Cheng, Y., Wong R Fau - Soo, Y. O. Y., Soo Yo Fau - Wong, W. S., Wong Ws Fau - Lee, C. K., Lee Ck Fau - Ng, M. H. L., Ng Mh Fau - Chan, P., . . . Cheng, G. (2020). Use of convalescent plasma therapy in SARS patients in Hong Kong. (0934-9723 (Print)).

Chersich, M. F., Gray, G., Fairlie, L., Eichbaum, Q., Mayhew, S., Allwood, B., . . . Rees, H. (2020). COVID-19 in Africa: care and protection for frontline healthcare workers. Global Health, 16 (1), 46. doi: 10.1186/s12992-020-00574-3

Commission., C. N. H. (accessed 2020 Apr 12). Chinese Clinical Guidance for COVID-19 Pneumonia Diagnosis and Treatment ( $\left.7^{\text {th }} \mathrm{ed}\right)$. fromhttp://kjfy.meetingchina.org/msite/news/show/cn/3337.html

Coondoo, A., Phiske, M., Verma, S., \& Lahiri, K. (2014). Side-effects of topical steroids: A long overdue revisit. Indian dermatology online journal, 5 (4), 416-425. doi: 10.4103/2229-5178.142483

Costanzo, M., De Giglio, M. A. R., \& Roviello, G. N. (2020). SARS-CoV-2: Recent Reports on Antiviral Therapies Based on Lopinavir/Ritonavir, Darunavir/Umifenovir, Hydroxychloroquine, Remdesivir, Favipiravir and Other Drugs for the Treatment of the New Coronavirus. LID 10.2174/0929867327666200416131117 [doi]. (1875-533X (Electronic)).

Cui, J. A.-O., Li, F., \& Shi, Z. A.-O. X. Origin and evolution of pathogenic coronaviruses. (1740-1534 (Electronic)).

Cullen, W., Gulati, G., \& Kelly, B. D. (2020). Mental health in the COVID-19 pandemic. QJM, 113 (5), 311-312. doi: 10.1093/qjmed/hcaa110

de Wit, E., Feldmann, F., Cronin, J., Jordan, R., Okumura, A., Thomas, T., . . . Feldmann, H. (2020). Prophylactic and therapeutic remdesivir (GS-5734) treatment in the rhesus macaque model of MERS-CoV infection. Proc Natl Acad Sci U S A, 117 (12), 6771-6776. doi: 10.1073/pnas.1922083117

Deng, S. Q., \& Peng, H. A.-O. (2020). Characteristics of and Public Health Responses to the Coronavirus Disease 2019 Outbreak in China. LID - 10.3390/jcm9020575 [doi] LID - 575. (2077-0383 (Print)).

Duan, K., Liu, B., Li, C., Zhang, H., Yu, T., Qu, J., . . . Yang, X. (2020). Effectiveness of convalescent plasma therapy in severe COVID-19 patients. Proc Natl Acad Sci U S A, 117 (17), 9490-9496. doi: $10.1073 /$ pnas. 2004168117

Eisenberg, J. (2020). R0: How Scientists Quantify the Intensity of an Outbreak Like Coronavirus and Its Pandemic Potential.

Elfiky, A. A. (2020). Anti-HCV, nucleotide inhibitors, repurposing against COVID-19. Life Sci, 248 , 117477. doi: $10.1016 /$ j.lfs.2020.117477

Epidemiology Working Group for Ncip Epidemic Response, C. C. f. D. C., \& Prevention. (2020). [The epidemiological characteristics of an outbreak of 2019 novel coronavirus diseases (COVID-19) in China]. Zhonghua Liu Xing Bing Xue Za Zhi, 41 (2), 145-151. doi: 10.3760/cma.j.issn.0254-6450.2020.02.003

Esposito, S., Noviello, S., \& Pagliano, P. (2020). Update on treatment of COVID-19: ongoing studies between promising and disappointing results. (1124-9390 (Print)).

Evans, A. S. (2013). Viral infections of humans: epidemiology and control : Springer Science \& Business Media.

Ferner, R. E., \& Aronson, J. K. (2020). Chloroquine and hydroxychloroquine in covid-19. Bmj, 369 (17561833 (Electronic)), m1432. doi: 10.1136/bmj.m1432 
Gamage, B., Moore, D., Copes, R., Yassi, A., Bryce, E., \& Group, B. C. I. R. P. S. (2005). Protecting health care workers from SARS and other respiratory pathogens: a review of the infection control literature. Am J Infect Control, 33 (2), 114-121. doi: 10.1016/j.ajic.2004.12.002

Gorbalenya, A. E. (2020). Severe acute respiratory syndrome-related coronavirus-The species and its viruses, a statement of the Coronavirus Study Group. BioRxiv .

Grein, J., Ohmagari, N., Shin, D., Diaz, G., Asperges, E., Castagna, A., . . . Flanigan, T. (2020). Compassionate Use of Remdesivir for Patients with Severe Covid-19. LID - 10.1056/NEJMoa2007016 [doi] LID - NEJMoa2007016. (1533-4406 (Electronic)).

Guan, W. J., Ni, Z. Y., Hu, Y., Liang, W. H., Ou, C. Q., He, J. X., . . . China Medical Treatment Expert Group for, C. (2020). Clinical Characteristics of Coronavirus Disease 2019 in China. N Engl J Med, 382 (18), 1708-1720. doi: 10.1056/NEJMoa2002032

Health., D.-G. f. (2020). COVID-19: mitigation phase. Approach of the patient with suspicion or infection with SARS-CoV-2., from https://www.dgs.pt/directrizes-da-dgs/normas-e-circulares-normativas/norma-n0042020-de-23032020-pdf

Huang, C., Wang, Y., Li, X., Ren, L., Zhao, J., Hu, Y., . . . Cao, B. (2020). Clinical features of patients infected with 2019 novel coronavirus in Wuhan, China. Lancet, 395 (10223), 497-506. doi: 10.1016/S01406736(20)30183-5

Huang, J. Z., Han, M. F., Luo, T. D., Ren, A. K., \& Zhou, X. P. (2020). [Mental health survey of medical staff in a tertiary infectious disease hospital for COVID-19]. Zhonghua Lao Dong Wei Sheng Zhi Ye Bing Za Zhi, 38 (3), 192-195. doi: 10.3760/cma.j.cn121094-20200219-00063

Imai, K., Tabata, S., Ikeda, M., Noguchi, S., Kitagawa, Y., Matuoka, M., . . . Maeda, T. Clinical evaluation of an immunochromatographic IgM/IgG antibody assay and chest computed tomography for the diagnosis of COVID-19. (1873-5967 (Electronic)).

Jakhar, D. A.-O., \& Kaur, I. (2020). Potential of chloroquine and hydroxychloroquine to treat COVID-19 causes fears of shortages among people with systemic lupus erythematosus. (1546-170X (Electronic)).

Jie, C., Fang, L., \& Zheng-Li, S. (2019). Origin and evolution of pathogenic coronaviruses. Nature reviews. Microbiology, 17 (3).

Jin, Y. H., Cai, L., Cheng, Z. S., Cheng, H., Deng, T., Fan, Y. P., . . . Health, C. (2020). A rapid advice guideline for the diagnosis and treatment of 2019 novel coronavirus (2019-nCoV) infected pneumonia (standard version). Mil Med Res, 7 (1), 4. doi: 10.1186/s40779-020-0233-6

Juurlink, D. N. (2020). Safety considerations with chloroquine, hydroxychloroquine and azithromycin in the management of SARS-CoV-2 infection. Cmaj, 192 (17), E450-E453. doi: 10.1503/cmaj.200528

Kang, S., Peng, W., Zhu, Y., Lu, S., Zhou, M., Lin, W., . . . Deng, M. (2020). Recent progress in understanding 2019 novel coronavirus (SARS-CoV-2) associated with human respiratory disease: detection, mechanisms and treatment. Int J Antimicrob Agents, 55 (5), 105950. doi: 10.1016/j.ijantimicag.2020.105950

Kaplan, S. S., \& Hicks, C. B. (2005). Lopinavir/ritonavir in the treatment of human immunodeficiency virus infection. Expert Opin Pharmacother, 6 (9), 1573-1585. doi: 10.1517/14656566.6.9.1573

Keeling, M. J., \& Rohani, P. (2011).Modeling infectious diseases in humans and animals: Princeton University Press.

Kim, C. J., Choi, W. S., Jung, Y., Kiem, S., Seol, H. Y., Woo, H. J., . . . Kim, Y. S. (2020). Surveillance of the MERS Coronavirus Infection in Healthcare Workers after Contact with Confirmed MERS Patients: Incidence and Risk Factors of MERS-CoV Seropositivity. Clinical Microbiology $\mathcal{E}$ Infection the Official Publication of the European Society of Clinical Microbiology 83 Infectious Diseases, S1198743X16302415. 
Kimberly E. Hanson, A. M. C., Cesar A. Arias. (2020). Infectious Diseases Society of America Guidelines on the Diagnosis of COVID-19.

Kisely, S., Warren, N., McMahon, L., Dalais, C., Henry, I., \& Siskind, D. (2020). Occurrence, prevention, and management of the psychological effects of emerging virus outbreaks on healthcare workers: rapid review and meta-analysis. Bmj, 369 (1756-1833 (Electronic)), m1642. doi: 10.1136/bmj.m1642

Kristensen, K. E., Torppedersen, C., Gislason, G. H., Egfjord, M., Rasmussen, H. B., \& Hansen, P. R. (2015). Angiotensin-Converting Enzyme Inhibitors and Angiotensin II Receptor Blockers in Patients With Abdominal Aortic AneurysmsSignificance.Arterioscler Thromb Vasc Biol, 35 (3), 733-740.

Kuba, K., Imai, Y., Rao, S., Gao, H., Guo, F., Guan, B., . . . Deng, W. (2020). A crucial role of angiotensin converting enzyme 2 (ACE2) in SARS coronavirus-induced lung injury.11 (8), 875-879.

Lee, B. Y. (2020). Study: 17.9\% Of People With COVID-19 Coronavirus Had No Symptoms.

Lentini, G. A.-O., Cavalluzzi, M. M., \& Habtemariam, S. A.-O. (2020). COVID-19, Chloroquine Repurposing, and Cardiac Safety Concern: Chirality Might Help. LID - E1834 [pii] LID - 10.3390/molecules25081834 [doi]. (1420-3049 (Electronic)).

Li-Li, R., Ye-Ming, W., Zhi-Qiang, W., Zi-Chun, X., Li, G., Teng, X., . . . Jian-Wei, W. (2020). Identification of a novel coronavirus causing severe pneumonia in human: a descriptive study. Chinese medical journal .

Li G Fau - De Clercq, E., \& De Clercq, E. Therapeutic options for the 2019 novel coronavirus (2019-nCoV). (1474-1784 (Electronic)).

Li G Fau - De Clercq, E., \& De Clercq, E. (2020). Therapeutic options for the 2019 novel coronavirus (2019-nCoV). (1474-1784 (Electronic)).

Li, W., Yang, Y., Liu, Z. H., Zhao, Y. J., Zhang, Q., Zhang, L., . . . Xiang, Y. T. (2020). Progression of Mental Health Services during the COVID-19 Outbreak in China. Int J Biol Sci, 16 (10), 1732-1738. doi: $10.7150 /$ ijbs. 45120

Li, Z., Yi, Y., Luo, X., Xiong, N., Liu, Y., Li, S., . . . Ye, F. (2020). Development and clinical application of a rapid IgM-IgG combined antibody test for SARS-CoV-2 infection diagnosis. LID - 10.1002/jmv.25727 [doi]. (1096-9071 (Electronic)).

Linton, N. M., Kobayashi, T., Yang, Y., Hayashi, K., Akhmetzhanov, A. R., Jung, S.-m., . . . Nishiura, H. (2020). Epidemiological characteristics of novel coronavirus infection: A statistical analysis of publicly available case data. medRxiv .

Luigi Angelo, V., Giovanni, S., \& Giacomo De, R. (2020). The importance of olfactory and gustatory disorders as early symptoms of coronavirus disease (COVID-19).British Journal of Oral and Maxillofacial Surgery .

Macau., U. o. (2020). UM professional team works with government to provide psychological counselling during epidemic period., from https: //wwwumedumo/news-centre/news-and-events/news-and-pressreleases/detail/49950/\#news_title (access February 17, 2020). 2020

Malta, M., Rimoin, A. W., \& Strathdee, S. A. (2020). The coronavirus 2019-nCoV epidemic: Is hindsight 20/20? (2589-5370 (Electronic)).

Marto, N., \& Monteiro, E. C. (2020). Medicines for the Treatment Of COVID-19: Awaiting the Evidence. LID - 10.20344/amp.13908 [doi]. (1646-0758 (Electronic)).

Medicine, N. H. C. S. A. o. T. C. (2020). Diagnosis and Treatment Protocol for Novel Coronavirus Pneumonia. 
medicine, P. S. o. c. (2020). Recommendations of the Portuguese Society of intensive care and group infection and sepsis on the method of covid-19., from https://www.spci.pt/media/covid-19/COVID_19_R_20200331.pdf.

Natsuko Imai, A. C., Ilaria Dorigatti, Marc Baguelin, Christl A. Donnelly, Steven Riley, Neil M. Ferguson. (2020). Report 3: Transmissibility of 2019-nCoV.

Nurses, T. I. C. o. (2020). ICN calls for data on healthcare worker infection rates and deaths.

P, W. A. C., Xin, L., P, L. S. K., \& Y, W. P. C. (2019). Global Epidemiology of Bat Coronaviruses. Viruses, 11 (2).

Peng, X., Xu, X., Li, Y., Cheng, L., Zhou, X., \& Ren, B. (2020). Transmission routes of 2019-nCoV and controls in dental practice. Int J Oral Sci, 12 (1), 9. doi: 10.1038/s41368-020-0075-9

Pereira, B. A.-O. (2020). Challenges and cares to promote rational use of chloroquine and hydroxychloroquine in the management of coronavirus disease 2019 (COVID-19) pandemic: a timely review. (1521-6950 (Electronic)).

Pranab, C., Nazia, N., Anup, A., Bhabatosh, D., \& Raman, R. G. (2020). The 2019 novel coronavirus disease (COVID-19) pandemic: A review of the current evidence. The Indian Journal of Medical Research .

Rempenault, C., Combe, B., Barnetche, T., Gaujoux-Viala, C., Lukas, C., Morel, J., \& Hua, C. (2020). Metabolic and cardiovascular benefits of hydroxychloroquine in patients with rheumatoid arthritis: a systematic review and meta-analysis. (1468-2060 (Electronic)).

Ren, L. L., Wang, Y. M., Wu, Z. Q., Xiang, Z. C., Guo, L., Xu, T., . . . Wang, J. W. (2020). Identification of a novel coronavirus causing severe pneumonia in human: a descriptive study. Chin Med J (Engl), 133 (9), 1015-1024. doi: 10.1097/CM9.0000000000000722

Rothan, H. A., \& Byrareddy, S. N. (2020). The epidemiology and pathogenesis of coronavirus disease (COVID-19) outbreak. J Autoimmun, 109 , 102433. doi: 10.1016/j.jaut.2020.102433

Savarino A Fau - Di Trani, L., Di Trani L Fau - Donatelli, I., Donatelli I Fau - Cauda, R., Cauda R Fau - Cassone, A., \& Cassone, A. (2020). New insights into the antiviral effects of chloroquine. (1473-3099 (Print)).

Schmid, M. B., \& Fontijn, J. (2020). COVID-19 in pregnant women. LID - S1473-3099(20)30175-4 [pii] LID - 10.1016/S1473-3099(20)30175-4 [doi] FAU - Schmid, Manuel B. (1474-4457 (Electronic)).

Shajahan, A., Supekar, N. T., Gleinich, A. S., \& Azadi, P. (2020). Deducing the N- and O- glycosylation profile of the spike protein of novel coronavirus SARS-CoV-2. LID - cwaa042 [pii] LID - 10.1093/glycob/cwaa042 [doi]. (1460-2423 (Electronic)).

Shen, C., Wang, Z., Zhao, F., Yang, Y., Li, J., Yuan, J., . . . Liu, L. (2020). Treatment of 5 Critically Ill Patients With COVID-19 With Convalescent Plasma.Jama (1538-3598 (Electronic)). doi: 10.1001/jama.2020.4783

Shen, K., Yang, Y., Wang, T., Zhao, D., Jiang, Y., Jin, R., . . . Lin, L. (2020). Diagnosis, treatment, and prevention of 2019 novel coronavirus infection in children: experts' consensus statement. World Journal of Pediatrics , 1-9.

Shigemura, J., Ursano, R. J., Morganstein, J. C., Kurosawa, M., \& Benedek, D. M. (2020). Public responses to the novel 2019 coronavirus (2019-nCoV) in Japan: Mental health consequences and target populations. Psychiatry Clin Neurosci, 74 (4), 281-282. doi: 10.1111/pcn.12988

World Health Organization declares Global Emergency: A review of the 2019 Novel Coronavirus (COVID-19) , (2020). 
Stower, H. (2020). Lopinavir-ritonavir in severe COVID-19. Nat Med, 26 (4), 465. doi: 10.1038/s41591020-0849-9

Sun, J., He, W. T., Wang, L., Lai, A., Ji, X., Zhai, X., . . . Su, S. (2020). COVID-19: Epidemiology, Evolution, and Cross-Disciplinary Perspectives. Trends Mol Med, 26 (5), 483-495. doi: 10.1016/j.molmed.2020.02.008

Sun, P., Lu, X., Xu, C., Sun, W., \& Pan, B. (2020). Understanding of COVID-19 based on current evidence. Journal of medical virology .

Sun, Z., Thilakavathy, K., Kumar, S. S., He, G., \& Liu, S. V. (2020). Potential Factors Influencing Repeated SARS Outbreaks in China. Int J Environ Res Public Health, 17 (5), 1633. doi: 10.3390/ijerph17051633

Surveillances, V. (2020). The Epidemiological Characteristics of an Outbreak of 2019 Novel Coronavirus Diseases (COVID-19)-China, 2020. fromhttp://weekly.chinacdc.cn/en/article/id

Tian, S., Hu, W., Niu, L., Liu, H., Xu, H., \& Xiao, S. Y. (2020). Pulmonary Pathology of Early-Phase 2019 Novel Coronavirus (COVID-19) Pneumonia in Two Patients With Lung Cancer.J Thorac Oncol, 15 (5), 700-704. doi: $10.1016 /$ j.jtho.2020.02.010

Tiwari, R., Dhama, K., Sharun, K., Iqbal Yatoo, M., Malik, Y. S., Singh, R., . . . RodriguezMorales, A. J. (2020). COVID-19: animals, veterinary and zoonotic links. Vet Q, 40 (1), 169-182. doi: $10.1080 / 01652176.2020 .1766725$

Udugama, B., Kadhiresan, P., Kozlowski, H. N., Malekjahani, A., Osborne, M., Li, V. Y. C., . . . Chan, W. C. W. (2020). Diagnosing COVID-19: The Disease and Tools for Detection. ACS Nano, 14 (4), 3822-3835. doi: $10.1021 /$ acsnano.0c02624

van Griensven, J., Edwards T Fau - de Lamballerie, X., de Lamballerie X Fau - Semple, M. G., Semple Mg Fau - Gallian, P., Gallian P Fau - Baize, S., Baize S Fau - Horby, P. W., . . . Haba, N. (2020). Evaluation of Convalescent Plasma for Ebola Virus Disease in Guinea. (1533-4406 (Electronic)).

Wang, D., Hu, B., Hu, C., Zhu, F., Liu, X., Zhang, J., . . . Xiong, Y. (2020). Clinical characteristics of 138 hospitalized patients with 2019 novel coronavirus-infected pneumonia in Wuhan, China. Jama .

Wang, D., Hu, B., Hu, C., Zhu, F., Liu, X., Zhang, J., . . . Peng, Z. (2020). Clinical Characteristics of 138 Hospitalized Patients With 2019 Novel Coronavirus-Infected Pneumonia in Wuhan, China. Jama (1538-3598 (Electronic)). doi: 10.1001/jama.2020.1585

Wang, H. J., Du, S. H., Yue, X., \& Chen, C. X. (2020). Review and Prospect of Pathological Features of Corona Virus Disease. Fa Yi Xue Za Zhi, 36 (1), 16-20. doi: 10.12116/j.issn.1004-5619.2020.01.004

Wang, M., Cao, R., Zhang, L., Yang, X., Liu, J., Xu, M., . . . Xiao, G. (2020). Remdesivir and chloroquine effectively inhibit the recently emerged novel coronavirus (2019-nCoV) in vitro. Cell Res, 30 (3), 269-271. doi: $10.1038 / \mathrm{s} 41422-020-0282-0$

Wang, Y., Zhang, D., Du, G., Du, R., Zhao, J., Jin, Y., . . . Wang, C. (2020). Remdesivir in adults with severe COVID-19: a randomised, double-blind, placebo-controlled, multicentre trial. Lancet, 395 (10236), 1569-1578. doi: 10.1016/S0140-6736(20)31022-9

Warren, T. K., Jordan, R., Lo, M. K., Ray, A. S., Mackman, R. L., Soloveva, V., . . . Bavari, S. (2020). Therapeutic efficacy of the small molecule GS-5734 against Ebola virus in rhesus monkeys. (1476-4687 (Electronic)).

WHO. (2020a). Coronavirus disease (COVID-19) Situation Report. from https://www.who.int/docs/defaultsource/coronaviruse/situation-reports/20200522-covid-19-sitrep-123.pdf?sfvrsn=5ad1bc3_4

WHO. (2020b). WHO launches global megatrial of the four most promising coronavirus 
treatments. from https://www.sciencemag.org/news/2020/03/who-launches-global-megatrial-four-mostpromising-coronavirus-treatments

Wichmann, D., Sperhake, J. P., Lütgehetmann, M., Steurer, S., Edler, C., Heinemann, A., . . Kluge, S. (2020). Autopsy Findings and Venous Thromboembolism in Patients With COVID-19. LID - 10.7326/M202003 [doi]. (1539-3704 (Electronic)).

Wilson KC, C. S., Bai C, Rello J. (2020). COVID-19: Interim guidance on management pending empirical evidence; from an American Thoracic Society led international task force. from https://www.thoracic.org/professionals/clinical-resources/disease-related-resources/covid-19-guidance.pdf

Wu, C., Chen, X., Cai, Y., Xia, J., Zhou, X., Xu, S., . . Song, Y. (2020). Risk Factors Associated With Acute Respiratory Distress Syndrome and Death in Patients With Coronavirus Disease 2019 Pneumonia in Wuhan, China. LID - 10.1001/jamainternmed.2020.0994 [doi] LID - e200994. (2168-6114 (Electronic)).

Xiaobo, Y., Yuan, Y., Jiqian, X., Huaqing, S., Jia'an, X., Hong, L., . . Department of Critical Care Medicine, R. H. o. W. U. W. C. (2020). Clinical course and outcomes of critically ill patients with SARS-CoV-2 pneumonia in Wuhan, China: a single-centered, retrospective, observational study. The Lancet Respiratory Medicine, 8 (5).

Xie, M., \& Chen, Q. (2020). Insight into 2019 novel coronavirus - An updated interim review and lessons from SARS-CoV and MERS-CoV. (1878-3511 (Electronic)).

Xu, X., Chen, P., Wang, J., Feng, J., Zhou, H., Li, X., . . Hao, P. (2020). Evolution of the novel coronavirus from the ongoing Wuhan outbreak and modeling of its spike protein for risk of human transmission. Sci China Life Sci, 63 (3), 457-460. doi: 10.1007/s11427-020-1637-5

Xu, X. W., Wu, X. X., Jiang, X. G., Xu, K. J., Ying, L. J., Ma, C. L., . . Li, L. J. (2020). Clinical findings in a group of patients infected with the 2019 novel coronavirus (SARS-Cov-2) outside of Wuhan, China: retrospective case series.Bmj, 368 , m606. doi: 10.1136/bmj.m606

Xu, Z., Shi, L., Wang, Y., Zhang, J., Huang, L., Zhang, C., . . Wang, F. S. (2020). Pathological findings of COVID-19 associated with acute respiratory distress syndrome. Lancet Respir Med, 8 (4), 420-422. doi: 10.1016/S2213-2600(20)30076-X

Yang, X., Yu, Y., Xu, J., Shu, H., Xia, J., Liu, H., . . Shang, Y. (2020). Clinical course and outcomes of critically ill patients with SARS-CoV-2 pneumonia in Wuhan, China: a single-centered, retrospective, observational study. Lancet Respir Med, 8 (5), 475-481. doi: 10.1016/S2213-2600(20)30079-5

Ying, L., A, G. A., Annelies, W.-S., \& Joacim, R. (2020). The reproductive number of COVID-19 is higher compared to SARS coronavirus. Journal of travel medicine .

Yu, P. (2020). A Familial Cluster of Infection Associated With the 2019 Novel Coronavirus Indicating Possible Person-to-Person Transmission During the Incubation Period. (1537-6613 (Electronic)).

Zha, L., Li, S., Pan, L., Tefsen, B., Li, Y., French, N., . . Villanueva, E. V. (2020). Corticosteroid treatment of patients with coronavirus disease 2019 (COVID-19).Med J Aust, 212 (9), 416-420. doi: 10.5694/mja2.50577

Zhang, W., Du, R. H., Li, B., Zheng, X. S., Yang, X. L., Hu, B., . . Zhou, P. (2020). Molecular and serological investigation of 2019-nCoV infected patients: implication of multiple shedding routes. Emerg Microbes Infect, 9 (1), 386-389. doi: 10.1080/22221751.2020.1729071

Zhu, N., Zhang, D., Wang, W., Li, X., Yang, B., Song, J., . . Tan, W. (2020). A Novel Coronavirus from Patients with Pneumonia in China, 2019. New England Journal of Medicine .

Zu, Z. Y., Jiang, M. D., Xu, P. P., Chen, W., Ni, Q. Q., Lu, G. M., \& Zhang, L. J. (2020). Coronavirus Disease 2019 (COVID-19): A Perspective from China. Radiology, 200490. doi: 10.1148/radiol.2020200490

Figure legend: 
Figure 1. a.The number of Daily emerged confirmed cases of COVID-19 in China and abroad.b. The number of cumulative confirmed cases of COVID-19 in China and abroad.c. The number of cumulative confirmed cases of COVID-19 in countries outside of China (Top 20). d. The number of cumulative death and the mortality rate of COVID-19 in China and abroad.
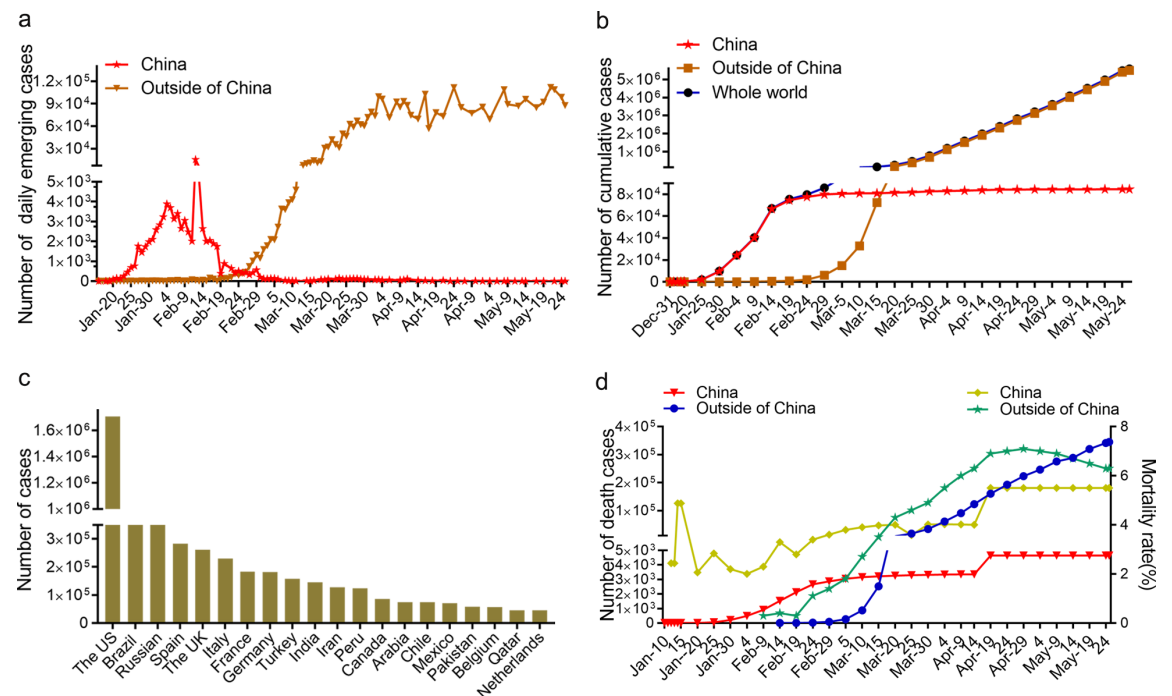

\section{Hosted file}

Table 1.docx available at https://authorea.com/users/328890/articles/456016-the-outbreak-ofcoronavirus-disease-2019-update-on-the-clinical-characteristics-and-treatment-strategies 